\title{
Flow Cytometry: Near Real-time INFORMATION ABOUT OCEAN BIOLOGY
}

\author{
By Clarice M. Yentsch
}

\begin{abstract}
A
LARGE FRACTION of planet Earth's photosynthesis is carried out by planktonic single-celled algae in the seas. Observation and delineation of regional and seasonal changes in plankton are major concerns of biological oceanography. Historically, the problem has been focussed on mapping these changes in the framework of seasonal and spatial change of ocean climatology. Early natural historians made elaborate drawings from astute observations of plankton. Recently, a major advance has come from the measurement of ocean color using Earth-orbiting satellites. The scientific basis for equating photosynthetic biology with ocean color is that the color change is due to the absorption of visible light by photosynthetic pigments of planktonic algae.
\end{abstract}

The Coastal Zone Color Scanner (CZCS) aboard the Nimbus satellite recorded changes in ocean color which depict how dynamic the photosynthetic process in the oceans is and how dependent this process is on the climatology and oceanography of various regions of the oceans. The observations made by the CZCS have provided a macro-mesoscale view of bulk distribution of phytoplankton. From these observations, it is apparent that there is a great deal of seasonal and spatial variability in the bulk properties of the phytoplankton. Yet, we are unable by this technique to ascertain how much of the variability is due to change in the type of particles absorbing light and how much is due to changes in the natural diversity of phytoplankton assemblages.

It is this change in diversity which is believed to be crucial to our understanding of climatological/ oceanographic influence and the global ocean photosynthetic process. Estimates of particle diversity require measurements at the scales opposite from satellite measurements; i.e., they require scaling down from kilometers to micrometers. At small scales, elaborate drawings of phytoplankton have been replaced by photomicrographs (Front cover). Epifluorescence microscopy has yielded even greater information on the diversity of living plankton (Fig. la, lb, p. 48). The beauty of the flow cytometry method outlined here is the possibility of measuring in real time individual characteristics of a large number of microscopic particles. The technique re-

Clarice M. Yentsch. J.J. MacIsaac Flow Cytometry/Cell Sorting Facility, Bigelow Laboratory for Ocean Sciences. West Boothbay Harbor, Maine 04575 lies on the diagnostic information obtained from single-cell fluorescence. The versatility of the interpretation is enhanced by corresponding measurements of particle volume and light scatter. The diagnostic mode is also enhanced by using membranepermeable dyes, which make it possible with flow cytometry to both characterize the type of particle and identify its related kinetic properties.

This paper presents a brief description of the technique and application of flow cytometry to the study of particles in the seas. An example is given to illustrate the usefulness of the method and to point out some of the limitations arising when the technique is used to study the temporal and spatial patterns of phytoplankton in the oceans.

\section{Flow Cytometry: What is it?}

Flow cytometry is the measurement of cells suspended in a fluid flow. In oceanography we expand this definition to include the measurement of other particles in the flow. The instruments include optical, fluid-flow and electronic systems. Cells are aligned in single file via laminar flow achieved in a sheath fluid under pressure. Common flow rates are $0.1 \mathrm{ml}$ $\mathrm{min}^{-1}$. Cells intersect an interrogation region which is illuminated by a coherent beam from one or two lasers (Fig. 2, p. 48) or a mercury lamp. To detect the fluorescence from the particle, the flow cytometer has several photomultiplier tubes. Detected intensities are digitized, giving gradients of intensity from 1 to 256 or 1 to 1024 . For calibration, fluorescent microspheres are passed through the system periodically, and if necessary instrument settings are readjusted.

Flow cytometers simultaneously measure several characteristics of individual cells in a flow stream. Unique aspects of the technique include: speed (analysis rates in excess of $10^{5}$ particles per second); multiple parametric measurements (typically two to three colors of fluorescence, cell volume, and light scatter); and size (submicron-including bacteria, prochlorophytes, cyanobacteria-to 200 micron in diameter). Precise quantification of fluorescence and light scatter are the result. The more sophisticated instruments have an ability to physically separate or "sort" particles of interest, which can be critical both for validation of the signal and for isolation of subpopulations for culture and further study. To separate particles, the laminar flow is divided into a stream of droplets by a piezoelectric transducer. A saline drop- 

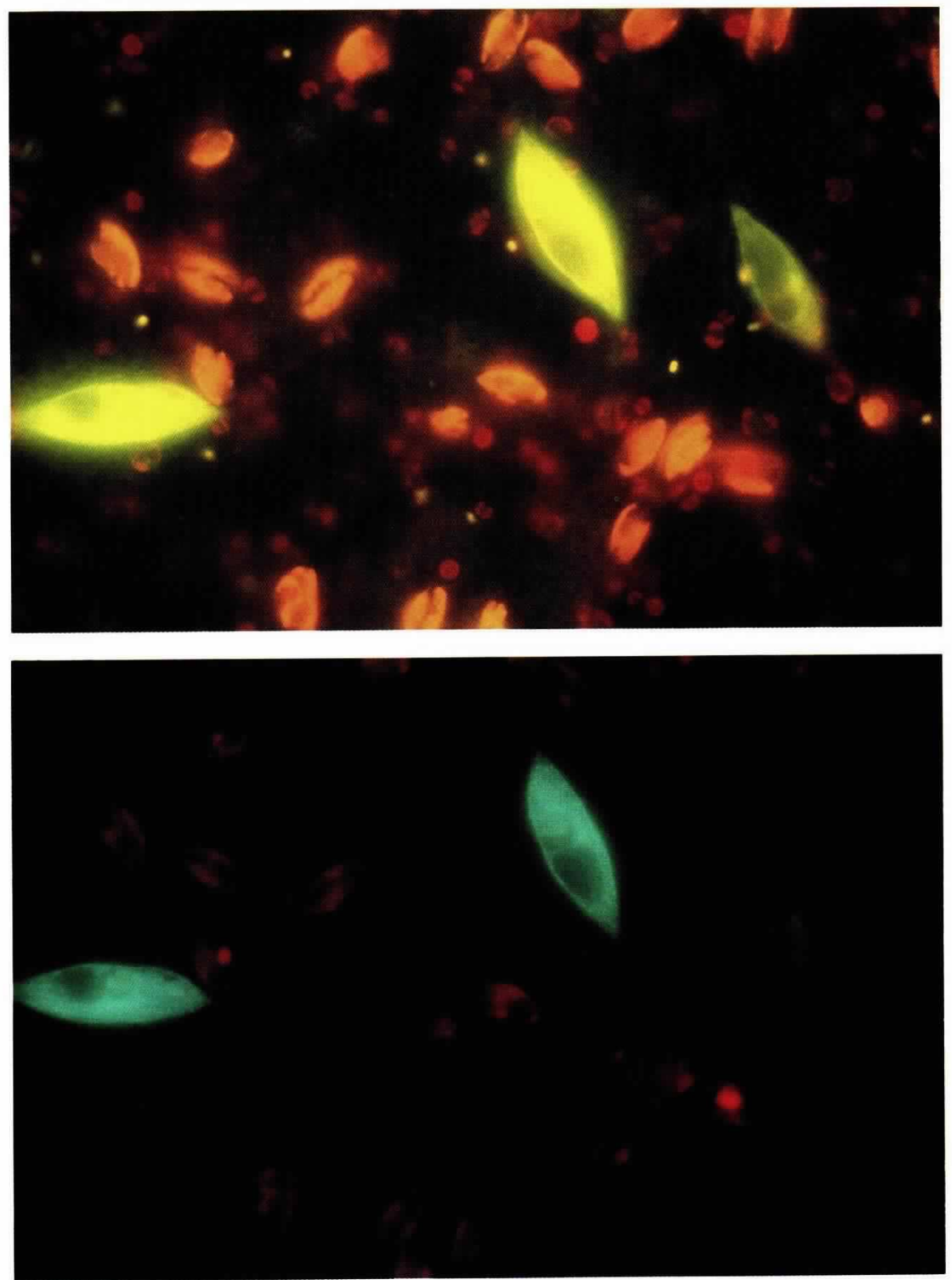

Fig. 1: Autofluorescent cells. (a, top): Epifluorescence micrograph of four plankton cultures depicting a medley of fluorescent colors using blue excitation, (b). Same field using ultraviolet excitation. (Photo courtesy of E. Haugen, Bigelow Laboratory, and L. Shapiro, University of Oregon).

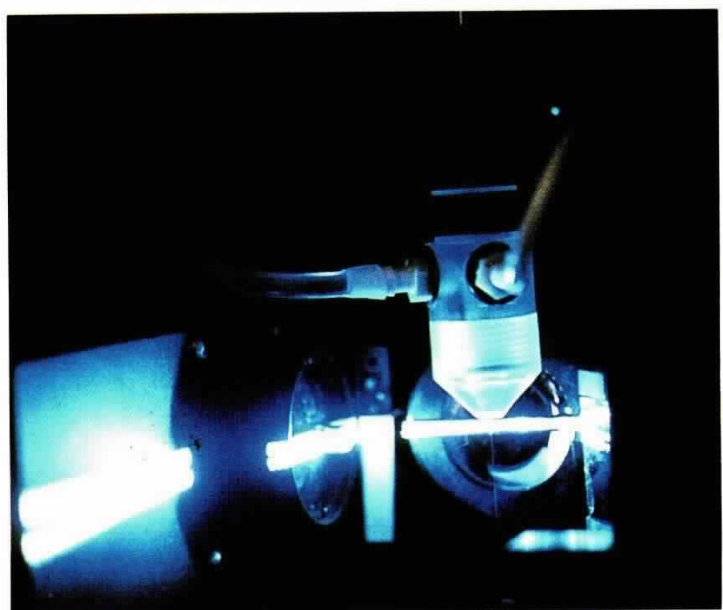

Fig. 2: Flow tip from Coulter EPICS V flow cytometer. Two argon-ion laser beams intersect cells in sample stream in the interrogation region. let containing the desired particle can be given an electrostatic charge and then moved left or right as it passes between high voltage deflection plates.

Without the property of autofluorescence from microorganisms, flow cytometry would not be a highly useful tool for oceanography. Fortunately, during the evolutionary course of photosynthesisapproximately 3.5 billion years-organisms developed antennae to capture sunlight. These antennae appear as pigment-protein complexes which absorb and fluoresce light in a manner similar to colored dyes, but which drive photosynthesis. The most commonly encountered photosynthetic pigments are chlorophyll- $a$ and phycoerythrin. Additionally there is a blue-green fluorescing pigment in microheterotrophs (Shapiro et al., 1988). Proper excitation transforms a mixture of several cell types and sizes into an array of colors of autofluorescence (See Fig. 1). Since these pigments absorb and fluoresce light, it is possible to exploit fluorescence for the study of phytoplankton (Yentsch and Yentsch, 1979; Legendre and Yentsch, 1989). As a result of such studies, a clear impression of the diversity of organisms responsible for ocean productivity is emerging for both new production and recycled production (Legendre and Le Fevre, 1989).

\section{Flow Cytometry: What can it do?}

One would suspect a sample of upper ocean water to contain suspended sediments, detritus (decaying organic matter), heterotrophic organisms and photosynthetic organisms. Flow cytometry can determine the relative fraction of any of these particles, and such determinations form the crux of research using the flow cytometer. For example, we know from our observations that the chlorophyll-bearing organisms rarely exceed $50 \%$ of the total number of particles which scatter light.

Representative sampling remains a concern. While bulk measurements and chemical measurements bias our interpretation toward larger cells with greater biovolume, flow cytometers bias our interpretations toward smaller particles. In one milliliter of seawater there are commonly $10^{6}$ bacteria and $10^{5}$ prochlorophytes and cyanobacteria, $10^{4}$ other small chlorophyll-containing cells, $10^{3}$ medium chlorophyll-containing cells and $10^{2}$ large-sized chlorophyll-containing cells, whereas larger cells such as ciliates might be present at one per ml. Accordingly, if we analyze only $0.1 \mathrm{ml}$, the likelihood of detecting a ciliate is one in ten and only ten large-sized chlorophyll-containing cells would be analyzed. Overcoming this limitation is important; thus faster flow rates at lower resolution and run times of several minutes versus seconds have been attempted.

\section{An Example}

An example of data resulting from measuring five parameters on $>10^{5}$ particles from a natural population in raw seawater is given in Fig. 3. The three colors of fluorescence emission, when excited at 488 $\mathrm{nm}$, include red fluorescence from chlorophyll (CHL $\mathrm{F}$; greater than $650 \mathrm{~nm}$ emission), orange fluores- 
cence from phycoerythrin (PE F; emission approximately $560-590 \mathrm{~nm}$ ) and blue-green fluorescence (emission approximately $515-530 \mathrm{~nm}$; the source of this can be autofluorescence from blue-green fluorescing microheterotrophs or from kinetic stains such as fluorescein diacetate, FDA). Two light scatter measurements were made: foward-angle light scatter (FALS - approximately 1-19 degrees) and side scatter (SSC-approximately 70-110 degrees). The data in Fig. 3 are displayed on four-decade log scales in the horizontal; the vertical axis shows particle count. The data are presented in two ways. First, we assign the particles into subpopulation clusters by fluorescence ataxonomy, grouping particles by similar particle type based on autofluorescence and cell size. Then the groupings are color-coded following a Becton-Dickinson algorithm which yields a six-color "dot plot," with each particle appearing as one dot of a specific color according to its classification. That classification holds throughout further analysis. Figure 3a shows the optical information, and Fig. 3b shows the biological information obtained by this technique. Subpopulations are interpreted from the dot plots, and six color-coded clusters are delineated: R1 (yellow) are small chlorophyll-containing cells; R2 (green) are detritus/microheterotrophs, with very low fluorescence for the particle size; R3 (red) are cryptomonads which have both chlorophyll and phycoerythrin-fluorescence; R4 (dark blue) are nonchlorophyll-containing particles; R5 (purple) are medium-sized chlorophyll-containing cells; and R6 (light blue) are large-sized chlorophyll-containing cells.

Figure 3 a shows forward-angle light scatter plotted against side scatter, both on four-decade log scales covering the range from 1 to 1024 channels. The number of particles measured gives the third (vertical) dimension. If light scatter properties alone are used, there can be no delineation as to whether particles are suspended sediment, detritus, microheterotrophs or photosynthetic autotrophs. The color coding results from gating based on corresponding autofluorescence analysis.

Stains are added to characterize and further distinguish the subpopulations of particles. For example, the stain FDA (fluorescein diacetate) can be used to distinguish different groups of particles on the basis of metabolic activity (Fig. 3b and Table 1, p. 50). A background control must be run for each stain assay. In the example presented, the background or natural abundance of the blue-green fluorescing cells is low (Table 1). For the purposes of this study, FDA or other stains serve primarily as agents to increase the number of parameters that can be used to distinguish subpopulations of particles and to characterize their dynamics. Note that in some subpopulations nearly all of the cells are positive or metabolically active. The cryptomonads, for example, are $99.9 \%$ metabolically active; this can be said with confidence. At low fluorescence detection levels however, the question is more complex; for example, further investiga-
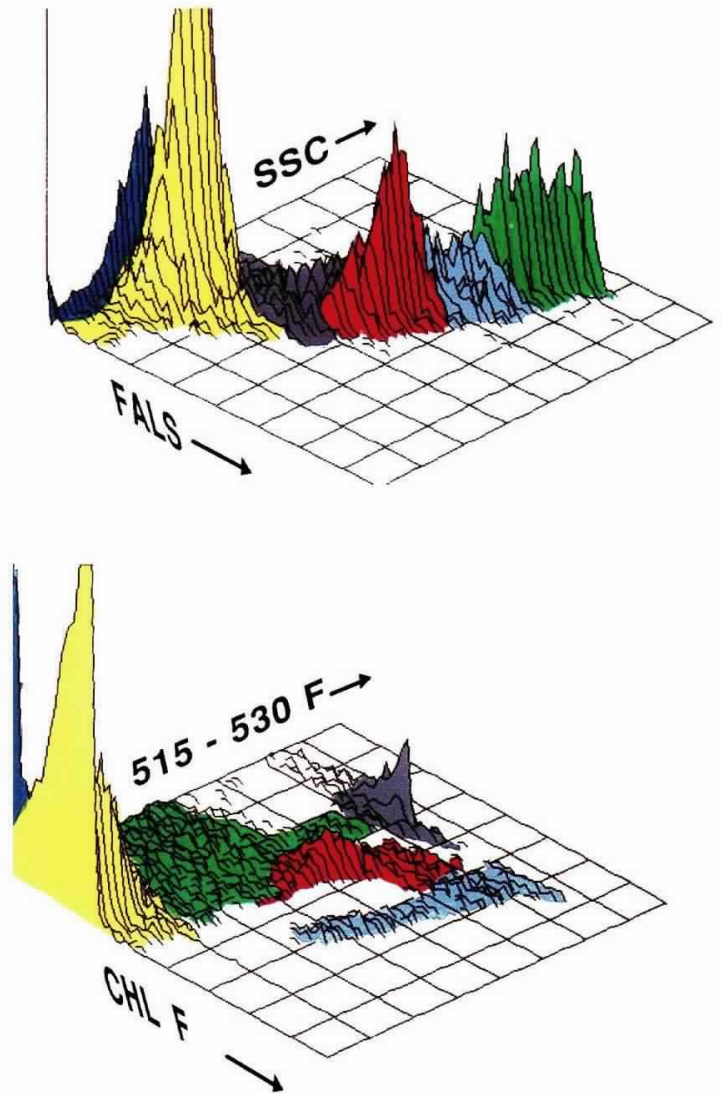

Fig. 3: Natural population segregated into taxa based on three color analysis. The sample, collected from the Sheepscot Estuary, Wiscasset, Maine, on June 21, 1990, was analyzed on a Becton-Dickson FACScan instrument. The figure shows an ataxonomic grouping into phycoerythrin-containing $(P E F)$ and chlorophyll-containing (CHLF) cells, cryptomonads (color-coded red), and three fluorescence intensity clusterings of chlorophyll-containing cells. (a, top): Optical data, showing side light scatter (SSC) versus forward angle light scatter (FALS). (b): Biological data for the same sample, showing CHL F versus blue-green fluorescence $(515-530 \mathrm{~nm})$, with fluorescein diacetate stain (FDA) added (ten minute exposure). Percentage positives of each subpopulation are presented in Table I. In both (a) and (b), the horizontal axes are four-decade log scales, and the vertical axes is linear cell count

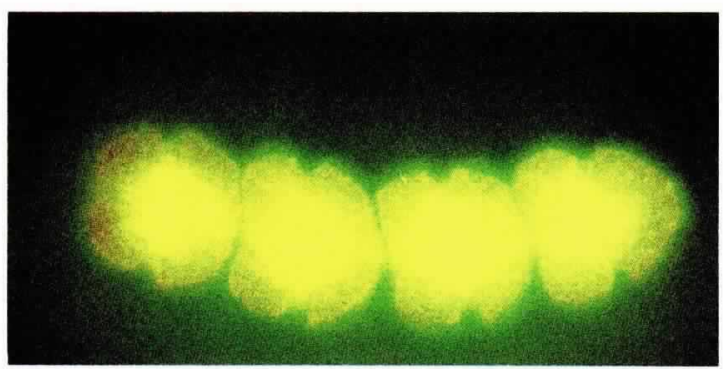

Fig. 4: Visualization of pattern of metabolic activity by the stain FDA of phytoplankton cells Gymnodinium catenatum. Cells are positive and remain actively swimming (Photo: K. Yeatts and C.M. Yentsch, Bowdoin College and Bigelow Laboratory). 


\section{Table 1}

Total number of particles $(\mathrm{N})$ : number falling above threshold for "positives" $515-530 \mathrm{~nm}$ emission (\#) and percentage (\%) of cells positive for natural population without FDA added (left) with FDA stain added (right) for six subpopulations based on ataxonomic clusters of chlorophyll- $a$ fluorescence and phycoeyrthrin fluorescence. Subpopulations are: R1 (yellow)—small chlorophyll-containing cells: R2 (green)-detritus/microheterotrophs-very low fluorescence for size; R3 (red)—cryptomononads having both chlorophyll and phycoerythrin; R4 (dark blue)—non-chlorophyll-containing particles; R5 (purple)—medium chlorophyllcontaining cells; R6 (light blue)—large chlorophyll-containing cells.

\begin{tabular}{|c|c|c|c|c|c|c|}
\hline & \multicolumn{3}{|c|}{$\begin{array}{l}\text { Natural Population } \\
\text { Without Stain }\end{array}$} & \multicolumn{3}{|c|}{$\begin{array}{l}\text { Natural Population } \\
\text { With Stain Added }\end{array}$} \\
\hline & $\mathrm{N}$ & $\#$ & $\%$ & $\mathrm{~N}$ & $\#$ & $\%$ \\
\hline R1 & 4163 & 0 & 0 & 4033 & 40 & 0.99 \\
\hline $\mathrm{R} 2$ & 1783 & 36 & 2.00 & 1588 & 507 & 31.92 \\
\hline R3 & 833 & 5 & 0.60 & 766 & 765 & 99.90 \\
\hline $\mathrm{R} 4$ & 2034 & 0 & 0 & 2113 & 321 & 15.19 \\
\hline R5 & 1091 & 0 & 0 & 1253 & 736 & 58.73 \\
\hline R6 & 435 & 15 & 3.44 & 515 & 492 & 95.50 \\
\hline
\end{tabular}

tion is needed to determine whether the small chlorophyll-fluorescing cells are inactive or merely below the levels of sensitivity. Microscopic observations are of limited usefulness here, as the flow cytometer is far more sensitive than the human eye. Figure 4 (p. 49 ) is a photomicrograph depicting FDA-positive cells in a chain of four dinoflagellate cells, following the method of Dorsey et al. (1989). The FDA-positive cells remain actively swimming. Note the similarity in color to the blue-green fluorescing cells of Fig. 1a. While running the "background/control" for this assay the abundance of these autofluorescent microheterotrophs in the Gulf of California and many other regions of the world's oceans was highlighted.

The example shown in Fig. 3 is based on measurements of autofluorescence of chlorophyll, phycoerythrin, blue-green fluorescence, plus forward angle and side light scatter, and a cytochemical kinetic probe, FDA. Additional measurements can be made using product "probes" to determine concentration of lipids and proteins, immunochemical probes (e.g. Shapiro et al., 1989 and the review by Ward, 1990), and oligonucleotide probes. The flow cytometrist's approach to such measurements is to ask "What can we measure on or inside the cell membrane?" whereas the oceanographer's approach is to ask "What can this tell us about physical, chemical or biological processes active outside the membrane?"

\section{Summary}

At last we are able to provide near real-time biological and optical information at sea in a format compatible with physical and chemical data. Flow cytometry provides an increasingly important part of the information base on ocean biology and particle optics, with special attention to the primary producers and microfoodweb components. When coupled with other biological data, such as bioluminescence via bathyphotometry, total particles via transmissometry, and zooplankton abundance via acoustic imaging/acoustic doppler current profilers (ADCP), measurements from flow cytometers bring within reach the hope of interweaving data and disciplines.

\section{Acknowledgements}

Thanks to G. Allison, J. Campbell, P. Colby, T. Cucci, J. J. Cullen, E. Haugen, C. Joule, D. Phinney and C. S. Yentsch of Bigelow Laboratory; L. Shapiro of the Oregon Institute of Marine Biology, University of Oregon; D. Gebhardt, L. Rhodes and K. Watson of Becton-Dickinson; and J. Logan, Research Publicist.

\section{References}

Dorsey. J., C.M. Yentsch. C. McKenna and S. Mayo, 1989: Rapid analytical technique for assessment of metabolic activity. Cytometry. 10, 622-628

Legendre. L. and J. Le Fevre, 1989: Hydrodynamical singularities as controls of recycled versus export production in oceans. In: Productivity of the ocean: Present and past. W.H. Berger. V.S. Smetacek, G. Wefer, editors, John Wiley \& Sons. Chichester, England, 49-63.

and C.M. Yentsch, 1989: Overview of flow cytometry and image analysis in biological oceanography and limnology. Cytometry. 10, 501-510.

Shapiro, L.P., E.M. Haugen and E.J. Carpenter, 1988: Occurrence and abundance of green-fluorescing dinoflagellates in surface waters of the Northwest Atlantic and Northeast Pacific Oceans. J. Phycol. 25. 189-191.

, L. Campbell and E.M. Haugen, 1989: Immunochemical recognition of phytoplankton species. Mar. Ecol. Prog. Ser. 57, 219-224.

Ward, B.B., 1990: Immunology in biological oceanography and marine ecology. Oceanogr.. 3(I), 30-35.

Yentsch, C.S. and C.M. Yentsch, 1979: Fluorescence spectral signatures: The characterization of phytoplankton populations by the use of excitation and emission spectra. J. Mar. Res. 37, 471-483. $\square$ 